\title{
Nuevos hábitos de consumo audiovisual en menores: aproximación a su análisis mediante encuestas
}

\section{New audiovisual consumption habits among minors: approximation through the analysis of survey data}
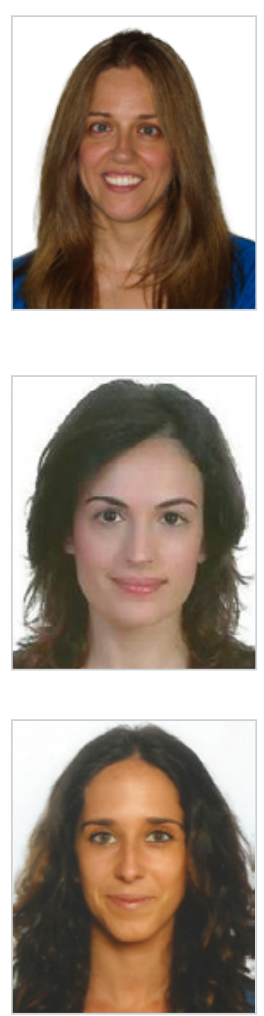

María José García-Orta. Licenciada y Doctora en Periodismo por la Universidad de Sevilla. Es profesora asociada en la Facultad de Comunicación de Sevilla y docente en el Máster en Comunicación Institucional y Política de dicha Universidad. Además, es miembro del grupo de investigación Medios, Políticas de Comunicación y Democracia en la Unión Europea. Su actividad científica la ha desarrollado tanto en el ámbito de la propaganda, como en otras parcelas de conocimiento, entre las que destacan la comunicación política electoral, la estructura de la información, la industria audiovisual y las nuevas tecnologías de la información, con especial atención a las redes sociales.

Universidad de Sevilla, España

mjorta@us.es

ORCID: 0000-0002-3284-2005

Victoria García-Prieto' Contratada predoctoral PIF por la Universidad de Sevilla. Licenciada en Periodismo (2010), Máster en Comunicación y Cultura (2012) y Técnico Superior en Interpretación de la Lengua de Signos Española (2015). Investigadora visitante de la Universidad de Cambridge (2018) y del Communication and Media Research Institute (CAMRI) de la Universidad de Westminster (2016 y 2017) y miembro del grupo de investigación Medios, Políticas de Comunicación y Democracia en la Unión Europea.

Universidad de Sevilla, España

vgarcia8@us.es

ORCID: 0000-0003-4973-7583

Miriam Suárez-Romero². Contratada predoctoral FPU del Ministerio de Educación. Máster en Comunicación Institucional y Política y licenciada en Periodismo por la Universidad de Sevilla. Estudia el tratamiento del liderazgo político femenino en los medios de comunicación. Miembro del grupo de investigación Medios, Políticas de Comunicación y Democracia en la Unión Europea (DEMOC-MEDIA).

Universidad de Sevilla, España

miriamsuarez@us.es

ORCID: 0000-0003-3672-7917

1 Este trabajo ha sido posible gracias a la financiación del Plan Propio de la Universidad de Sevilla.

2 Trabajo predoctoral financiado por el Ministerio de Educación, Cultura y Deporte para la Formación del Profesorado Universitario.

Cómo citar este artículo:

García-Orta, M. J.; García-Prieto, V.; Suárez-Romero, M. (2019). Nuevos hábitos de consumo audiovisual en menores: aproximación a su análisis mediante encuestas. Doxa Comunicación, 28, pp. 241-260.

https://doi.org/10.31921/doxacom.n28a13 
Recibido: 18/11/2018 - Aceptado: 08/05/2019

\section{Resumen:}

La llegada de Netflix en 2015 propició que otros grupos de comunicación tuvieran presencia en España a través de plataformas online. La situación se aceleró en 2016 y comenzó a observarse una tendencia clara hacia el consumo de contenidos no lineales. Por ello, este trabajo tiene como objetivos principales conocer el consumo audiovisual de los jóvenes entre 14 y 17 años y estudiar el rol de los usuarios en estas plataformas.

Se utiliza una metodología estructural basada en: revisión bibliográfica, estudios exploratorios para determinar estos cambios y encuestas a alumnos de ESO y Bachillerato. Los resultados permiten determinar cuáles son los patrones de consumo de los menores, qué géneros predominan, qué plataformas utilizan más y si son prosumidores de contenidos.

\section{Palabras clave:}

Vulnerabilidad digital, vídeo bajo demanda, televisión no lineal consumo audiovisual, plataformas OTT.
Received: 18/11/2018 - Accepted: 08/05/2019

\section{Abstract:}

The arrival of Netflix in 2015 promoted the presence of other communication groups in Spain through online platforms. The situation accelerated in 2016 and a clear tendency toward the consumption of non-linear content could be observed. Therefore, the main objectives of this study are to gain knowledge regarding the audiovisual consumption of young people between 14 and 17 years of age and to study the role of users on these platforms. A structural methodology has been used that is based on the following: a bibliographic review, exploratory studies to determine these changes, and surveys of students of lower and upper Secondary Educacion. The results of this research have allowed us to determine the consumption patterns of minors, the genres that predominate, the platforms they use most, and whether they are content prosumers

\section{Keywords:}

Digital vulnerability, video on demand, non-linear television, audiovisual consumption, OTT platforms.

\section{Introducción}

Hace trece años Peñafiel, Fernández de Arroyabe y López (2005: 125), expresaban que “la audiencia cada vez está más preparada para codificar los mensajes que recibe y romper con el sistema unilateral de recepción que ha reinado en nuestra televisión". A pesar del tiempo transcurrido, esta afirmación está más vigente que nunca. El vaticinio de diversos académicos en fases primigenias de este nuevo acontecer se ha transformado ahora en una realidad consolidada. Hoy, el consumo audiovisual no se limita a la televisión lineal, pues se ha producido un cambio en los hábitos de utilización de los contenidos de vídeo, como pone de manifiesto el Informe Anual del Sector de los Contenidos Digitales en España (Muñoz López, 2017), que indica que la suscripción (Subscription Video On Demand, SVOD) se ha consolidado desde 2015 como el principal modelo de consumo de este tipo de contenidos.

Así, según señala Izquierdo Castillo (2017):

(...) los cambios derivados de la convergencia tecnológica sobre la televisión lineal obligan a reubicarla como un medio de distribución en combinación con otras plataformas, principalmente internet (Caldwell, 2006; Creeber y Hills, 2007; Meikle y Young, 2008), lo que afecta directamente al contenido, que se concibe desde el origen con una estrategia de distribución de 360 grados, en combinación con otras plataformas (Parker, 2007). (...) también supone la desvinculación del contenido respecto a un medio y, lo que es más significativo, respecto a una industria (p. 32).

El desarrollo del vídeo bajo demanda (VOD) ha sido posible, según Clares-Gavilán y Medina-Cambrón (2018: 911), gracias a cambios estructurales y coyunturales, junto con una serie de factores de alta influencia asociados. Es el caso, por ejemplo, 
de la implantación de la banda ancha, el desarrollo de nuevos dispositivos tecnológicos, el uso de televisiones inteligentes (Smart TVs) o el desarrollo de contenidos para consumo en casa, entre otros.

Gran parte de la eclosión del sistema está relacionada con el consumo de series. Con base en un estudio realizado por Kantar Media (Garza, 2017, online) comenta: "El consumo de series de televisión se extiende cada vez más alrededor del mundo. En España el 50\% de la población con acceso a internet consume series de televisión en la red”. Además, asistimos a una consolidación de esta tendencia, con unos consumidores que "demandan cada vez más un servicio de televisión a la carta, ofrecido por estas plataformas y adecuado a sus necesidades, puesto que un $30 \%$ de ellos declaran utilizar este tipo de servicios para crear sus propios horarios de programas de televisión" (Garza, 2017).

Onieva (2017, online) resalta que cada español dedica una media de tres horas diarias al visionado de televisión pero, no obstante, las formas de consumo son distintas, pues "cuatro de cada diez españoles ve, al menos una vez a la semana, contenidos online. Y parte de la culpa de este fenómeno la tienen las plataformas de vídeo bajo demanda como Movistar, Netflix y HBO España, cuyo número de suscriptores crece como la espuma".

Según las cifras de la CNMC (2018), la plataforma más utilizada a finales de 2017 fue Movistar+, con 2,16 millones de usuarios, lo que supone un 13,5\% de los hogares con conexión a Internet en España, duplicando casi los datos del año anterior. Le siguió Netflix con 1,5 millones de suscriptores o un 9,1\% de los hogares, triplicando las cifras en un año. En tercer lugar, se encuentran Vodafone TV online, con un 5,9\% de los hogares (944.000 suscriptores); Amazon Prime Video con el 3,5\% (566.000 suscriptores); Orange TV online con el 2,8\% (450.000 suscriptores) y en el sexto puesto HBO con el 2,3\% de los hogares (363.000 suscriptores). En definitiva, tres de cada diez hogares usaron servicios de pago para ver contenidos audiovisuales online a finales del pasado año (CNMC, 2018).

A la luz de las cifras, estamos ante una tesitura de cambio en los hábitos y rutinas de consumo de los usuarios a nivel general, en el contexto de "una sociedad siempre conectada y plenamente digitalizada, que se encuentra en pleno proceso de redefinición del cómo asigna su tiempo a la hora de consumir productos y servicios de los medios de comunicación" (Ortega, González Ispierto y Pérez Peláez, 2015: 643).

El móvil ha sido, sin duda, uno de los elementos disruptivos en este proceso transformador, "y derivado de la mejora del ancho de banda, de la concurrencia en los servidores audiovisuales, y por supuesto una clara [sic] evolución en los equipos de telefonía móvil, la visualización de contenidos audiovisuales se está desarrollando y asentando a pasos agigantados" (Caldera-Serrano y León-Moreno, 2017: 63).

También Adelantado y Martí (2011) analizaron las posibilidades del móvil como plataforma de difusión y consumo de contenidos audiovisuales. Pusieron de relevancia una de las mayores ventajas que ha traído aparejadas: "haber conquistado tiempos y espacios hasta el momento no adscritos al consumo mediático tradicional" (Adelantado y Martí, 2011: 102). Unos años después, Ortega, González Ispierto y Pérez Peláez (2015) destacaban que los “usos y consumos de los productos audiovisuales entre los jóvenes se trasladan progresivamente hacia canales de distribución interactivos ubicados en pantallas inteligentes individuales y personalizadas" (p. 643).

Con el rápido desarrollo de este soporte, además de las tabletas, la televisión no lineal ha conseguido un espacio propio que le ha robado a la televisión tradicional, con la que vive en coexistencia. De Mena Dávila (2015) opina que la segunda no 
desaparece, pero sí comparte protagonismo con otros dispositivos, con distintas pantallas. "Y eso es especialmente destacable en el caso de los usuarios más jóvenes, que a lo largo del día van cambiando de un dispositivo a otro para consumir contenido audiovisual (móvil, tableta, ordenador...)” (Mena Dávila, 2015: 36).

Son precisamente los jóvenes el objeto de estudio de esta investigación, protagonistas principales de los nuevos hábitos de consumo audiovisual. El informe de Limelight Networks (2017), “The State of Online Video”, apunta en esta dirección:

Online video viewing continues to grow. Consumers are increasingly taking advantage of the ability to watch what they want, on their own schedules. Globally, people who watch online video view an average of five hours and 45 minutes per week, up 34 percent in less than one year. For people age 18-25, that number already exceeds seven hours per week, with more than a quarter watching more than ten hours of online video each week.

(...) When analyzed by age, younger viewers watch more online video per week than older viewers, with people 18-25 watching 7.30 hours per week versus people over 60 watching 3.77 hours.

Estos datos pueden observarse en la siguiente tabla, donde la media de horas semanales (en la última columna) es mayor entre los jóvenes de 18 a 25 años.

Tabla 1. Horas semanales de visionado de vídeo online.

\begin{tabular}{|c|c|c|c|c|c|c|}
\hline Ages & $\begin{array}{c}1-2 \text { hours } \\
\text { per week }\end{array}$ & $\begin{array}{c}2-4 \text { hours } \\
\text { per week }\end{array}$ & $\begin{array}{c}4-7 \text { hours } \\
\text { per week }\end{array}$ & $\begin{array}{c}7-10 \text { hours } \\
\text { per week }\end{array}$ & $\begin{array}{c}\text { More than } \\
\text { 10 hours } \\
\text { per week }\end{array}$ & $\begin{array}{c}\text { Weighted Average } \\
\text { Viowing Timo } \\
\text { (Hours/Week) }\end{array}$ \\
\hline $\mathbf{1 8 - 2 5}$ & $14.8 \%$ & $21.2 \%$ & $23.0 \%$ & $15.3 \%$ & $25.9 \%$ & $\mathbf{7 . 3 0}$ \\
\hline $\mathbf{2 6 - 3 5}$ & $18.2 \%$ & $21.2 \%$ & $21.7 \%$ & $16.3 \%$ & $22.7 \%$ & $\mathbf{6 . 8 8}$ \\
\hline $\mathbf{3 6 - 4 5}$ & $24.3 \%$ & $23.9 \%$ & $20.8 \%$ & $14.0 \%$ & $17.1 \%$ & $\mathbf{5 . 9 7}$ \\
\hline $\mathbf{4 6 - 6 0}$ & $39.8 \%$ & $25.1 \%$ & $13.7 \%$ & $9.8 \%$ & $11.6 \%$ & 4.68 \\
\hline $\mathbf{6 0 +}$ & $51.9 \%$ & $23.0 \%$ & $10.6 \%$ & $7.0 \%$ & $7.5 \%$ & 3.77 \\
\hline
\end{tabular}

Fuente: Limelight Networks (2017).

Los datos sobre los hábitos que recoge este informe están extraídos de las respuestas de mayores de edad de Francia, Alemania, India, Filipinas, Singapur, Corea del Sur, Reino Unido y Estados Unidos. ¿Y en España? Según la Comisión Nacional de los Mercados y la Competencia (CNMC, 2018) los jóvenes entre 16 y 24 años consumen más contenido audiovisual a través de su dispositivo móvil que de la televisión, con 4,7 horas diarias entre ordenador, tableta y móvil, frente a las 2,2 de la televisión.

También Vidales, Aldea y De la Viña (2012) estudiaron el consumo de televisión en jóvenes, específicamente de aquellos con edades entre los 14 y los 25 años, "el segmento poblacional que menos televisión consume en la actualidad. En España, su índice ha descendido por debajo de los 10 puntos, pasando de un $20 \%$ en 1995, por ejemplo, a un estimativo $6 \%$ según los últimos estudios de TNS y el EGM de 2009" (p. 104). Las autoras señalan, en la línea del resto de estudios, que las preferencias de los jóvenes se inclinan hacia los contenidos bajo demanda y las visualizaciones en ordenadores y móviles de todo tipo de contenidos distribuidos por estos medios. Y extraen valiosas conclusiones de su investigación: los jóvenes 
creen que la televisión no está hecha para ellos, sino para adultos, criticando el lenguaje que usan (inadecuado para jóvenes y niños), sin respetar el horario infantil, sin información veraz e incluso sin ética ni profesionalidad. "La radiografía presentada podemos resumirla en la siguiente frase: han perdido el interés” (Vidales, Aldea y De la Viña, 2012: 111).

Por su parte, Guerrero Pérez (2018) ha examinado recientemente la evolución que ha seguido el consumo de televisión lineal de los millennials, comparándolo con el resto de la audiencia sin segmentar:

Los sectores más jóvenes de la población-millennialsy generaciones venideras de menor edad-muestran un comportamiento audiovisual que prioriza los servicios siempre conectados y personalizables, frente a las ofertas rígidas y cerradas. Se sienten atraídos por aquellos productos que les otorgan un papel activo, no solo como consumidores, sino también como programadores y productores, situándoles en el núcleo de las historias que les interesan (Guerrero Pérez, 2018: 1.243).

El autor marca el punto de inflexión en 2013, cuando "el consumo televisivo lineal desciende de modo progresivo y generalizado hasta la actualidad, resultando especialmente acusado entre los públicos más jóvenes. En solo cinco años, el visionado de televisión se redujo en 21 minutos de media por persona y día" (Guerrero Pérez, 2018: 1.234), un descenso que, según este autor, coincide con el auge de los servicios de catch up de las televisiones tradicionales, que a través de sus plataformas online permiten al usuario visualizar contenidos ya emitidos por la cadena de forma lineal, así como productos producidos exclusivamente para la plataforma.

Guerrero Pérez (2018) destaca, además, dos años claves: 2014, con la eclosión de la televisión de pago en España por su inclusión en los paquetes combinados (móvil, fijo, internet) que los operadores ofertaban (p. 1235); y 2015, momento en el que Netflix entra en el mercado español del vídeo bajo demanda. Esta plataforma ha sabido cambiar las reglas del mercado televisivo y cinematográfico, ya que no sólo se dedica a la distribución de obras, sino que se ha lanzado a la producción de series. "Un jugador que entendió rápidamente que su negocio aprovecha la convergencia tecnológica y mediática (Heredia, 2017: 284).

En 2016 se le unirían HBO España y Amazon Prime Video, y un año más tarde Sky. Todas ellas plataformas internacionales que ampliaron la oferta audiovisual que ya ofrecían plataformas SVOD como Filmin y Rakuten TV (antes Wuaki TV). El sector del vídeo se completa con servicios de AVOD (Advertisement Video on Demand), que ofrecen al usuario acceso gratuito e ilimitado a su catálogo a cambio de la inserción de publicidad, como es el caso de YouTube. "En consecuencia, el mercado español pasó, en tan solo unos años, de un estado de escasez a una situación de sobreoferta audiovisual" (Guerrero Pérez, 2018: 1.235).

Una oferta en la que el espectador ya no está atado a los horarios de las cadenas, sino que "puede optar por visionar los contenidos cuando lo desee bien grabándolos o viéndolos en streaming” (Quintas y González, 2016: 380), configurando asî un consumo personalizado.

Esta tendencia ha ido aumentando progresivamente con el paso del tiempo. "La televisión sucumbe a Internet de la mano de los más jóvenes" era el titular elegido por Libre Mercado (Vega, 2018). Lo explican como sigue:

Las causas se encuentran principalmente en el cambio del comportamiento de los consumidores. Como publica World

Economic Forum, la renovación generacional es la clave para entender la sustitución de los medios en el país americano.

Mientras los mayores de 50 años se sientan frente el televisor durante una media de seis horas diarias (un aumento del $6 \%$

enero-junio de 2019

doxa.comunicación | n² 28, pp. 241-260 | 245 
desde el 2012), los jóvenes de entre 18 y 24 años dedican menos de dos horas al día al consumo de contenido televisivo (una caída del $44 \%$ con respecto a hace cinco años).

Cabe preguntarse a qué se deben estos cambios en los hábitos de consumo de los jóvenes. González Aldea y López Vidales (2011) consideran que está relacionado "con los nuevos soportes, la multiplicación de 'pantallas' y la aparición de un nuevo tipo de contenidos: contenidos fragmentados para audiencias fragmentadas. Es el fenómeno de la 'youtubización de la televisión'” (p. 37), donde predomina entre los jóvenes el consumo de vídeos de información y de entretenimiento. Su resumen es: contenidos diferentes para dispositivos diferentes.

Sánchez Vilela (2016, online) reflexiona sobre cómo el visionado de televisión es una marca de identidad, "un proceso de construcción de estilo de vida diferenciado”, y lo describe como sigue: “(...) la domesticación en los nuevos modos de ver televisión de los jóvenes implica decisiones en una doble articulación: la elección de dispositivos que permiten la movilidad y la elección de programas/ contenidos televisivos específicos que se insertan en la tarea de construcción de su vida cotidiana".

Los estudios, como hemos visto, ponen de relieve una evolución en el consumo marcada por una clara tendencia entre los jóvenes a consumir más contenidos audiovisuales a través de plataformas OTT (Over-The-Top) que televisión lineal o tradicional. ise debe al cambio generacional? Galán Fajardo y Del Pino Romero (2009) recuerdan cómo es frecuente escuchar afirmaciones estereotipadas sobre que los jóvenes de hoy son "muy diferentes a los de generaciones anteriores; y en gran parte esa enunciación es cierta, pues la sociedad en la que viven esos jóvenes no es la misma, ni tampoco lo es el sistema político y económico imperante" (p. 4) o, en el caso que nos atañe, la tecnología de la que disponen.

Teniendo todo esto en cuenta, la aproximación que presentamos aspira a aportar nuevos datos en la investigación de esta tendencia de cambio entre los jóvenes, concretamente en menores de edad, habida cuenta del condicionamiento innegable que supone en los modos y medios de creación, producción y distribución de los productos audiovisuales dirigidos a un público que consume de una forma determinada, y cuyas características pretendemos describir a pequeña escala.

\section{Metodología}

Todo lo expuesto supone la base teórica y los antecedentes necesarios para realizar este estudio que tiene como último objetivo conocer el consumo audiovisual que realizan los jóvenes de 14 a 17 años. Asimismo, nos planteamos en esta investigación conocer el rol que desempeñan los menores de edad en las plataformas online de vídeo, especialmente su papel como prosumidores, así como contextualizar los cambios de consumo y reflexionar sobre las posibles oportunidades y/o riesgos de este nuevo modelo.

Para ello, al inicio de la investigación se han planteado las siguientes cuestiones: ¿Cuál es la situación actual del consumo audiovisual en España?; ¿Por qué éste ha evolucionado hacia el entorno digital?; ¿Qué tipo de contenidos ven los menores en las plataformas online?; i Se asemeja su consumo al de los jóvenes entre 18 y 25 años, donde la ficción, en concreto las series de televisión, es la gran protagonista?; ¿Qué grado de interacción tienen los menores cuando consumen vídeos o contenidos audiovisuales de forma online?; $i$ El consumo bajo demanda es complementario o sustituye al lineal? 
Todas estas preguntas son la base de dos hipótesis:

H1. El consumo de contenidos audiovisuales en plataformas online por parte de menores se centra en el entretenimiento, asumiendo todavía un rol pasivo en el proceso de comunicación.

H2. Los menores podrían estar consumiendo contenidos culturales no adecuados para su franja de edad, concretamente series de ficción, sin el control pertinente por parte de los agentes implicados.

Para alcanzar los objetivos fijados y las hipótesis de trabajo, se han empleado dos etapas dentro de la investigación cualitativa: la investigación documental y las encuestas. En la primera etapa, la recogida de información se ha realizado a través de documentación bibliográfica, especialmente la referida a comportamientos del consumidor de contenidos audiovisuales, artículos en revistas del sector, así como diferentes estudios o informes relacionados con el consumo televisivo en España.

Con respecto a la segunda etapa, se ha optado por la encuesta como técnica de investigación más eficaz para alcanzar nuestros objetivos. En este sentido, la encuesta, gracias a procedimientos estandarizados, permite recoger y analizar datos de una muestra de casos representativa de una población más amplia, para así describir y/o explicar una serie de características (García, 1993). Esta técnica encaja perfectamente en el tipo de método utilizado en este trabajo, un método descriptivo transversal o de prevalencia, ya que analizamos la frecuencia del consumo audiovisual en el momento actual. Para ello, se ha recurrido a un muestreo aleatorio simple. Dado que el estudio es una aproximación a los cambios de consumo, se fijó inicialmente la realización de 100 cuestionarios a estudiantes con edades comprendidas entre los 14 y 17 años, aunque finalmente nuestra muestra ha sido de 111 menores. Para tener acceso a ese sector de la población, seleccionamos un instituto de enseñanza secundaria que tuviera niveles educativos de ESO y Bachillerato. El centro elegido, fundamentalmente por criterios de proximidad, fue el I.E.S. Néstor Almendros, del municipio sevillano de Tomares. Se trata de un centro con más de 1.700 alumnos, con 23 grupos de ESO y 12 grupos de Bachillerato (cuatro de ellos para adultos), un grupo de enseñanza secundaria para personas adultas (modalidad a distancia) y 18 grupos de formación profesional.

El siguiente paso en la investigación fue el diseño del cuestionario, donde se optó por combinar preguntas de respuesta fija, con otras de elección múltiple (abanico de respuestas con un ítem abierto) y preguntas abiertas. El cuestionario se diseñó a través de la plataforma estadounidense SurveyMonkey.

Después de contactar con la dirección del centro educativo, se estableció que los cuestionarios se hicieran impresos utilizando como intermediarios a los tutores de los grupos de $4^{\circ} \mathrm{de} \mathrm{ESO}$ y $1^{\circ}$ de Bachillerato, ya que eran los niveles educativos que se ajustaban por franjas de edad a nuestro estudio. La encuesta se realizó a un total de 8 grupos (con una media de 30 alumnos por clase) la semana del 18 al 22 de junio, coincidiendo con el final de curso, periodo que presentó una dificultad añadida: el absentismo de muchos alumnos. Esto provocó que se tuviera que repetir la encuesta en dos grupos de cuarto de ESO la tercera semana de septiembre. Además, en las clases de $1^{\circ}$ de Bachillerato había un alto índice de estudiantes mayores de edad, por lo que ellos no pudieron responder al cuestionario.

En definitiva, se ha diseñado una metodología estructural y crítica basada en una revisión bibliográfica para establecer el contexto y en estudios exploratorios a través de encuestas.

A continuación, se ofrece el cuestionario utilizado y diseñado para esta investigación. 
Nuevos hábitos de consumo audiovisual en menores: aproximación a su análisis mediante encuestas

Tabla 1. Cuestionario Jóvenes y hábitos de consumo en Internet

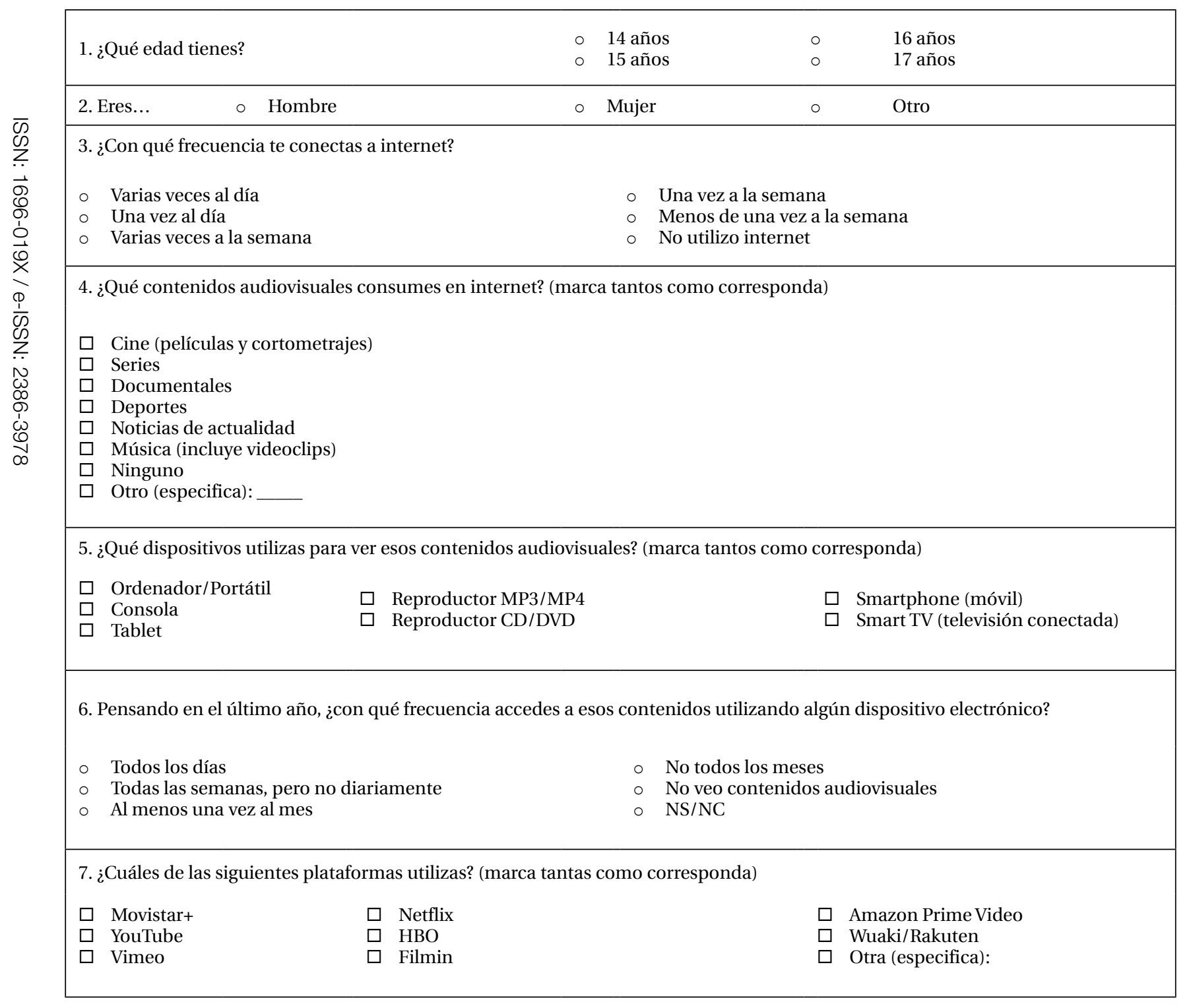


8. Cuando utilizas esas plataformas...

Me limito a ver los contenidos, pero no participo

Además de ver los contenidos, participo activamente (con comentarios, valorando el contenido...)

No las utilizo

9. ¿Has subido contenido audiovisual a internet?

No

- Sí (Especifica a qué plataforma):

10. ¿Sueles ver o has visto alguna vez contenidos audiovisuales para mayores de 18 años a través de internet?

- Sí, frecuentemente

Sí, pero ocasionalmente

- No, nunca

- NS/NC

11. ¿Qué piensan tus padres de los contenidos audiovisuales que es?

- Saben lo que veo y están de acuerdo

- Saben lo que veo, pero no están de acuerdo y lo veo sin su permiso

No saben lo que veo, pero si lo supiesen estarían de acuerdo

- No saben lo que veo y, si lo supiesen, no estarían de acuerdo

12. ¿Ves series de televisión o películas a través de páginas web gratuitas (e ilegales)?
○ Sí
$\circ \quad$ No
$\circ$
NS/NC

13. ¿Cómo consumes las series? (marca tantas como corresponda)

$\square$ En su emisión en televisión

$\square$ A la carta en la web del canal de televisión (Ej.: Atresplayer)

$\square \quad$ Las descargo de internet

$\square \quad$ En DVD o Blu-ray

$\square$ En plataformas gratuitas (como YouTube, Daily Motion o Vimeo)

$\square$ En un paquete de contenidos (como Netflix, HBO, Filmin o Movistar+)

14. ¿Cuántas series ves actualmente?:

15. ¿Cuántos capítulos de series ves a la semana?

16. De las series que ves o has visto, ¿cuáles son tus cinco favoritas?:

17. ¿Qué series prefieres?

- Series españolas

○ Series extranjeras

Fuente: Elaboración propia 


\section{Resultados}

A continuación, exponemos los resultados más destacables, siguiendo el orden del cuestionario recién presentado.

Las dos primeras preguntas de la encuesta iban encaminadas a establecer la edad y el sexo de los jóvenes encuestados. En cuanto a la edad, de los 111 participantes, 7 tenían 14 años, 39 tenían 15, 29 de ellos tenían 16 años, y 33 de los participantes tenían 17. Tres de ellos no contestaron correctamente a la pregunta, por lo que no ha podido clasificarse su edad. La siguiente gráfica muestra visualmente la distribución de los participantes por edad:

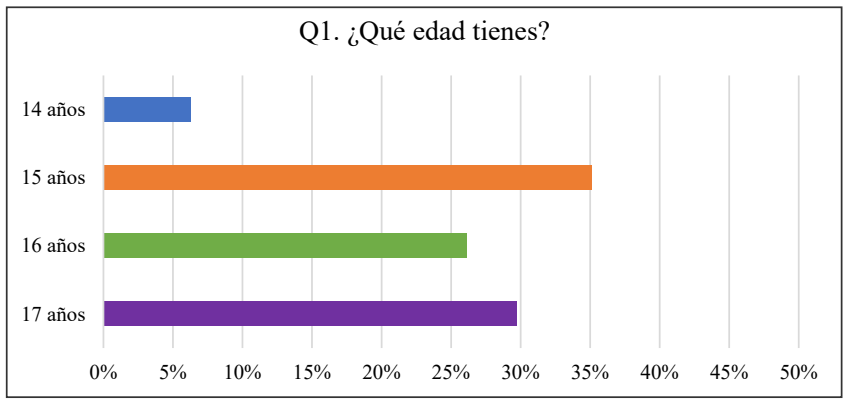

Fuente: Elaboración propia a partir de datos de encuestas realizadas utilizando la herramienta SurveyMonkey (en adelante, SM)

En cuanto al sexo, 61 de los participantes eran hombres (51,45\%), mientras que 48 eran mujeres (43,64\%). Solo una persona escogió la tercera opción ("otro") y otra no respondió a la pregunta correctamente, por lo que su respuesta fue descartada.

La tercera pregunta medía la frecuencia con la que los participantes se conectan a internet y 102 de los 111 encuestados afirmaron hacerlo varias veces al día. Esto supone más de un 93\%, y quienes no han escogido esta opción es porque se conectan una vez al día (3 personas) o varias veces a la semana (4 personas). Ninguno de los participantes ha dicho conectarse con menos frecuencia o no utilizar internet. Esto demuestra la alta penetración del uso regular de internet por parte de los jóvenes encuestados.

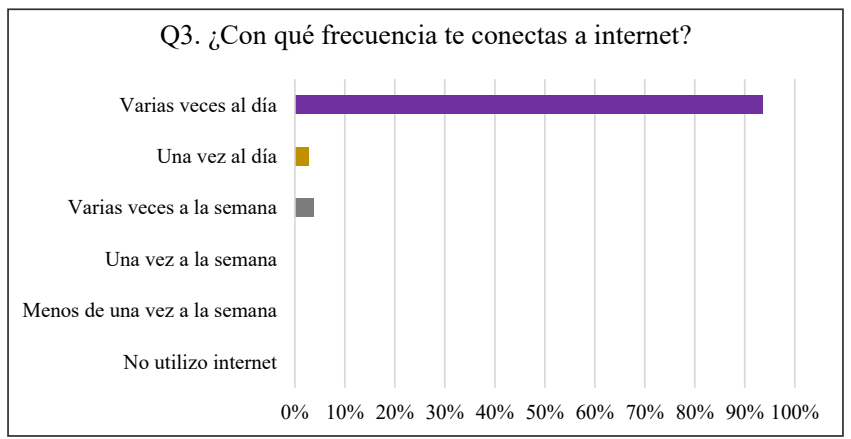

Fuente: propia a partir de datos de encuestas realizadas utilizando la herramienta SM. 
Una vez establecida la frecuencia de conexión a internet, la siguiente pregunta medía qué contenidos audiovisuales veían los participantes.

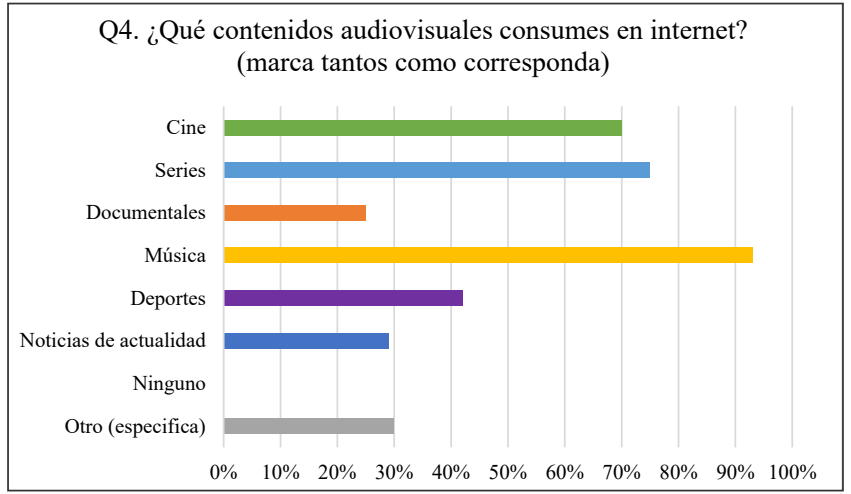

Fuente: propia a partir de datos de encuestas realizadas utilizando la herramienta SM.

Como se aprecia en la gráfica, más del 93\% de los encuestados afirma ver vídeos musicales y a este tipo de contenidos le siguen las series (75,68\%) y el cine (70,27\%). Además, ninguno de ellos escogió la opción "Ninguno", lo que confirma que todos los participantes consumen contenidos audiovisuales en internet. Por otra parte, algunas personas dijeron consumir otro tipo de contenidos como "videojuegos", "vídeos de YouTube”, "Instagram” u otro tipo de programas como “realities”. La respuesta más llamativa es la de tres participantes varones que dijeron consumir "pornografía” online.

En cuanto al dispositivo que utilizan para ver estos contenidos, el móvil es el claro ganador con un $90 \%$ de usuarios entre los encuestados, seguido del ordenador o portátil (76,58\%) y las Smart TV (54,95\%). Otras opciones como las consolas y los reproductores MP3/Mp4 o de DVD se mostraron más minoritarias.

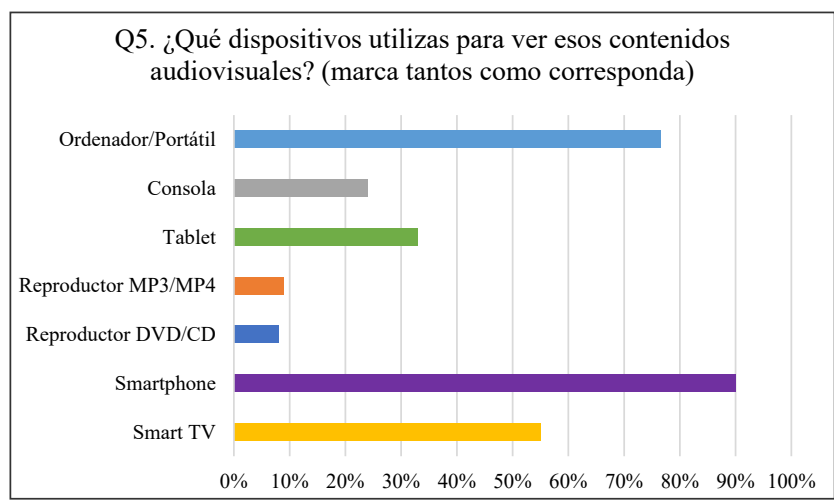

Fuente: propia a partir de datos de encuestas realizadas utilizando la herramienta SM. 
En la pregunta 6 se evaluaba no ya la frecuencia de acceso a internet, sino la frecuencia de consumo de los contenidos audiovisuales que decían ver. La pregunta era "Pensando en el último año, ¿icon qué frecuencia accedes a esos contenidos utilizando algún dispositivo electrónico?” y el 83,49\% de los participantes dijo hacerlo “todos los días”. Solo un 15\% dijo ver los contenidos "todas las semanas, pero no diariamente" y solo una persona $(0,92 \%)$ dijo hacerlo "mínimo una vez al mes". Frecuencias menores como "No todos los meses" o respuestas como "No veo contenidos audiovisuales" no fueron escogidas por ningún encuestado.

Adentrándonos más en el consumo audiovisual de los encuestados, la siguiente pregunta trataba sobre las plataformas que usan para ver los contenidos. La gran triunfadora es YouTube, utilizada por 110 de los 111 participantes (99,10\%). Le siguen, aunque con diferencia, Netflix (64,86\%), Movistar+ (39,64\%) y HBO (27,03\%). Otras opciones como Amazon Prime Video, Rakuten o Vimeo resultaron ser más minoritarias. Además, casi un 30\% de los participantes dijeron utilizar otras plataformas como "Plusdede", “Masdede”, "Spotify”, “AnimeFLV”, “AnimeYT”, “Repelis” o “Twitch TV”.

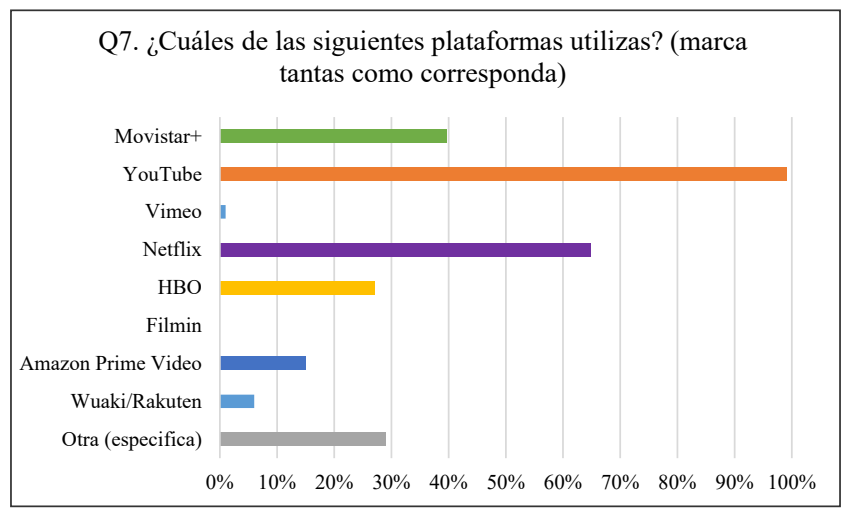

Fuente: propia a partir de datos de encuestas realizadas utilizando la herramienta SM.

A continuación, la pregunta 8 intentaba determinar si los participantes se limitaban a consumir contenidos audiovisuales o si, además, participaban activamente valorando o comentando los contenidos. A esta pregunta el 78,18\% respondió diciendo que se limitaba a ver los contenidos y solo el 20,91\% dijo participar activamente.

Del mismo modo, la pregunta 9 continuaba evaluando el rol activo de los participantes. En este caso se preguntaba: “ $i$ Has subido contenido audiovisual a internet?” De los 111 encuestados, 72 dijeron que no $(64,86 \%)$, mientras que 39 respondieron afirmativamente $(35,14 \%)$. A estos últimos se les pidió que especificaran a qué plataforma habían subido el contenido y las respuestas fueron: "Instagram”, “Twitter”, “Twitch TV”, "YouTube”, "Vimeo", "Facebook” y “SoundCloud”.

Adentrándonos aún más en el tipo de contenidos que ven los encuestados, la siguiente cuestión era: “¿Sueles ver o has visto alguna vez contenidos clasificados para mayores de 18 años a través de internet?” En esta encuesta realizada a 111 menores de entre 14 y 17 años, 84 de los participantes (75,68\%) dijeron ver contenidos para mayores de 18 años ya fuera frecuentemente u ocasionalmente. Por el contrario, solo un 16,36\% afirma no hacerlo nunca. 


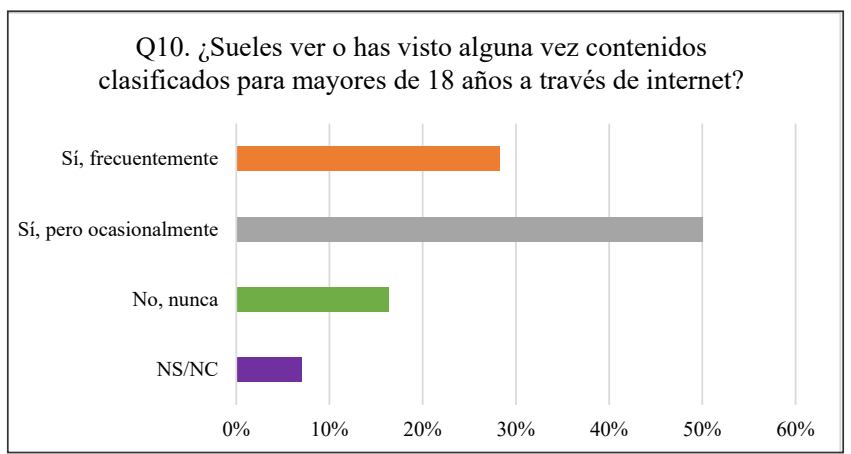

Fuente: propia a partir de datos de encuestas realizadas utilizando la herramienta SM.

Además, también se les preguntó qué pensaban sus padres de los contenidos que consumían en internet. Las opciones eran: 1) "Saben lo que veo y están de acuerdo", 2) "Saben lo que veo, pero no están de acuerdo y lo veo sin su permiso", 3) "No saben lo que veo, pero si lo supiesen estarían de acuerdo" y 4) "No saben lo que veo y, si lo supiesen, no estarían de acuerdo”. El 66\% afirma que sus padres conocen los contenidos que consumen y están de acuerdo con que los vean. Sin embargo, 37 afirman que sus padres no saben lo que ven en internet (33,33\%), aunque 32 de ellos dicen que, si lo supieran, estarían de acuerdo. Ninguno de ellos escogió la opción "Saben lo que veo, pero no están de acuerdo y lo veo sin su permiso".

También queríamos conocer si veían series o películas online en páginas web gratuitas e ilegales. En la pregunta 12, que se refería a esta cuestión, el 74,77\% de los encuestados reconocía que sí lo hacía, mientras que el 23,42\% negaba hacerlo.

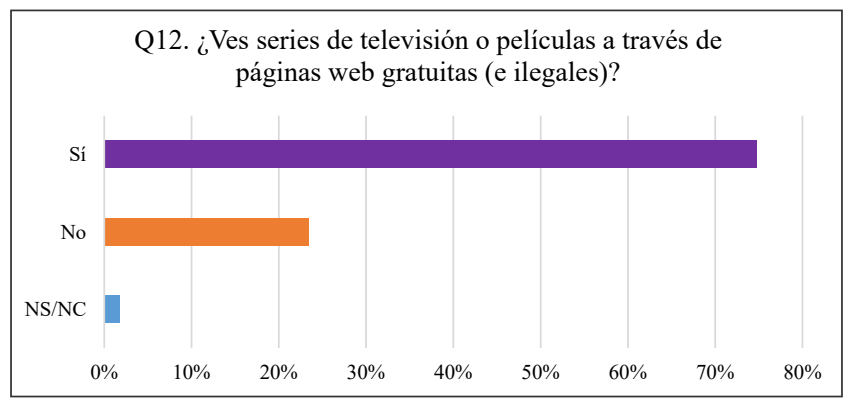

Fuente: propia a partir de datos de encuestas realizadas utilizando la herramienta SM.

Las siguientes preguntas se centraban exclusivamente en el consumo de series, ya que es uno de los contenidos más visto por los jóvenes en internet (el segundo tras los vídeos musicales en nuestra encuesta). Así, la pregunta 13 se encabezaba: “¿Cómo consumes las series?” Era una pregunta de respuesta múltiple y las opciones eran: 1) En su emisión en televisión; 2) 
A la carta en la web del canal de televisión (Ej.: Atresplayer); 3) Las descargo de internet; 4) En DVD o Blu-ray; 5) En plataformas gratuitas (como YouTube, Daily Motion o Vimeo); 6) En un paquete de contenidos (Netflix, HBO, Filmin o Movistar+).

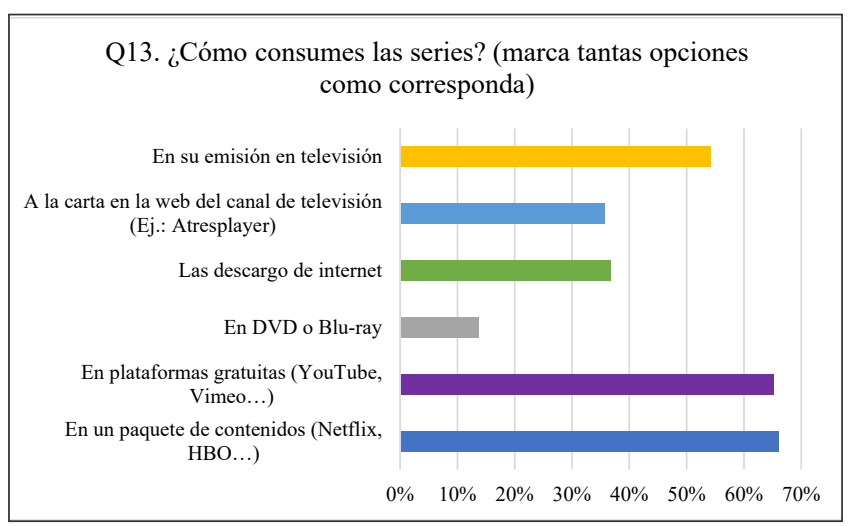

Fuente: propia a partir de datos de encuestas realizadas utilizando la herramienta SM.

Como se puede observar en la gráfica, las opciones más comunes son: "En un paquete de contenido” (66,06\%), "En plataformas gratuitas” (65,14\%) y “En su emisión en televisión” (54,13\%). Por el contrario, solo el 13,76\% consume las series en DVD o Blu-ray.

La siguiente cuestión planteada era una pregunta abierta sobre el número de series que consumen los encuestados. Las opciones más frecuentes son 0 y 1, con 26 respuestas cada una. Otros 16 participantes dicen ver 3 series en el momento de realización de la encuesta, 14 dicen ver 2 series y 9 reconocen ver 4 series cuando rellenaron la encuesta. Otros encuestados dicen ver entre 5 y 7 series y tres de ellos afirman ver más de 10 series al mismo tiempo.

Siguiendo un orden lógico, la pregunta 15 se refería a la cantidad de capítulos que veían a la semana, y también era una pregunta abierta. Para clasificar los resultados los hemos agrupado en varios intervalos: 1) 0 capítulos; 1) 1-5 capítulos; 2 ) 6-10 capítulos; 3) 11-15 capítulos; 4) 16 capítulos o más. Aunque 14 participantes dijeron no ver ningún capítulo de serie, la mayor parte de las respuestas se sitúa en el intervalo de 1-5 capítulos por semana (36 respuestas), seguido de 6-10 capítulos por semana (23 respuestas). 6 de los participantes vería entre 11 y 15 capítulos y 8 de ellos verían más de 16 capítulos semanales. Otras respuestas recopiladas no han podido cuantificarse, como, por ejemplo: "Los que pueda, porque me engancho"; "Pocos"; "Bastantes”; "Depende del tiempo, puedo verme una temporada diaria o nada en toda la semana”; "Ahora ninguno, más adelante”; “Si está en emisión, 1 a la semana. Si no, 5-20”.

Adentrándonos aún más en el consumo de series, en la pregunta 16 pedíamos a los participantes que nos dijeran cuáles eran sus cinco series favoritas. El siguiente listado contiene aquellas que han sido nombradas 10 o más veces:

1. Stranger Things (18 respuestas)

2. Juego de Tronos (15 respuestas)

3. La que se avecina (12 respuestas) 
4. Breaking bad (11 respuestas)

5. La casa de papel (11 respuestas)

6. Por trece razones (11 respuestas)

7. Rick y Morty (11 respuestas)

La mayoría de ellas tienen una calificación para mayores de 16 años, en algunos casos por su alto contenido de violencia y sexo.

Por último, concluía la encuesta con una pregunta cerrada sobre las preferencias de los encuestados entre series españolas y series extranjeras. El 81,73\% de los participantes dijo preferir las series extranjeras, mientras que las españolas solo fueron elegidas por el $18,27 \%$.

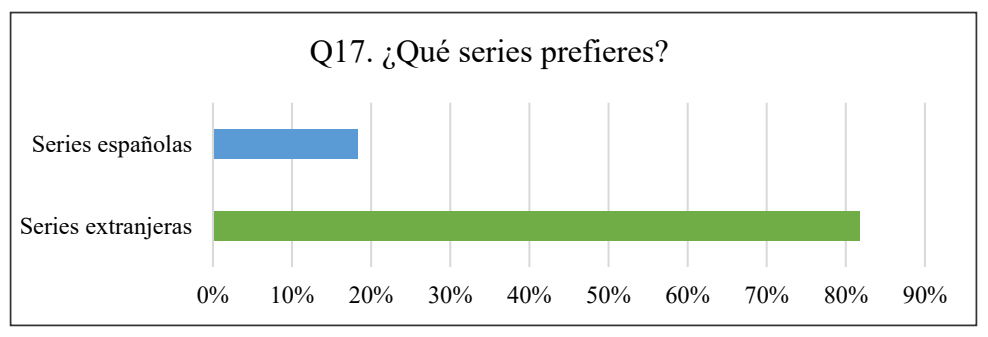

Fuente: propia a partir de datos de encuestas realizadas utilizando la herramienta SM.

\section{Discusión y conclusiones}

A grandes rasgos, podemos afirmar que los menores participantes en este estudio no siguen el ritmo de las parrillas clásicas de programación, ya que consumen preferentemente contenidos audiovisuales en internet, sobre todo de entretenimiento, y se conectan con bastante frecuencia, ya que más de un $93 \%$ acceden a la red varias veces al día, el mismo porcentaje que reconoce ver vídeos musicales.

Esta primera conclusión confirma que, en este caso, aunque se trate de un estudio muy concreto, específico y no representativo estadísticamente, también se demuestra el alto uso de internet por parte de los jóvenes, un comportamiento expuesto en la introducción teórica del artículo y sostenida por diversos académicos. La reflexión de Sánchez-Labella Martín (2015: 97) sobre la forma de relación con los medios es trasladable a lo obtenido:

La televisión, desde su nacimiento, ha sido el medio que mayor índice de audiencias ha captado, pero la aparición de Internet ha traído consigo la implantación de un nuevo ecosistema mediático revolucionando todo lo establecido hasta ahora en el ámbito de la ecología de los medios; así como el modo en que los individuos nos relacionamos con éstos. (...) Como en su momento apuntara Mcluhan "el contenido de cualquier medio o vehículo es siempre otro medio o vehículo" (Mcluhan, 1969, p. 22), pero Internet supera esta realidad: más que el contenido del medio anterior incluye los contenidos de todos los medios antecedentes. Es este factor el que justifica su éxito pues si "la hibridación de medios libera gran fuerza o energía 
como la fusión" (McLuhan, 1969, p. 67) cuanto más grande sea el número de formas que se funden más alta es la energía producida y, por tanto, mayor es el atractivo para los seres humanos (Canavilhas, 2011: 17).

Este atractivo al que se refiere la autora es también el observado a través de los resultados de este estudio en los menores participantes, con unos hábitos de consumo donde la ficción tiene un alto protagonismo, y en especial las series (75,68\% de los encuestados), tanto en VOD como en abierto en televisión lineal.

La mayoría de las series que consumen tienen una calificación para mayores de 16 años, en algunos casos por su alto contenido de violencia y sexo, como es el caso de Juego de Tronos, la segunda serie más vista por los encuestados. Estos datos plantean un debate sobre la aplicación efectiva de las nuevas normas de servicios de comunicación audiovisual de la Unión Europea (Consejo Europeo, 2018), donde se contempla que las plataformas online estarán obligadas a actuar para frenar el contenido que incite a la violencia y/o el odio.

Aunque este baremo de medición sólo es una recomendación no vinculante, el estudio pone en evidencia la falta de control parental, sobre todo en los jóvenes de 14 años, que confiesan ver series con una calificación para mayores de 16 años. Una ausencia de control que se pone de manifiesto cuando tres participantes varones reconocieron consumir "pornografía” online y el 75,68\% de los encuestados dijeron ver contenidos para mayores de 18 años.

Este problema social detectado en los resultados de las encuestas de este trabajo ha sido, sobre todo en los últimos años, objeto de investigación académica, alertando del incremento de su consumo entre jóvenes en edades muy tempranas. Un ejemplo interesante, en el contexto de Latinoamérica, es el estudio de Rivera, Santos, Cabrera y Docal (2016), que examina si los estilos de vida de los adolescentes son predictores relevantes de su consumo.

Por otro lado, a pesar de que los datos aseguran que las plataformas de VOD han reemplazado en buena medida a la piratería al ofrecer una tarifa plana asequible y un catálogo amplio, gran parte de los encuestados han confesado ver series o películas a través de páginas web gratuitas e ilegales, por lo que la piratería aún sigue siendo una cuestión vinculante. A la luz de los resultados de esta investigación, cabe cuestionarse esta teórica traslación hacia modelos de consumo de pago, pudiendo hablar más bien, y en este caso concreto, de una convivencia de herramientas digitales a la hora de consumir los productos seriados. También observamos cómo el consumo de vídeo bajo demanda convive con el consumo audiovisual tradicional, si bien la tendencia apunta hacia un paulatino desinterés por la televisión lineal y la sustitución progresiva de un medio por otro.

A pesar de que el segundo contenido más demandado en internet sean las series, en el momento de la encuesta 26 menores $(23,42 \%)$ reconocían no estar viendo ninguna serie y otros 26 sólo seguían una serie semanal. Sólo el 14,41\% reconocía ver tres series en el momento de realización de la encuesta. Aún así, el número de capítulos vistos por semana es bastante aceptable, ya que el 32,43\% ve entre 1-5 capítulos por semana; y el 20,72\% sigue de 6-10 capítulos semanales.

Podemos asimismo concluir que el entretenimiento es la gran apuesta de los jóvenes. Dentro de los contenidos que consumen se encuentran también los videojuegos, el contenido audiovisual a través de redes sociales como Instagram y algunos realities. La inmensa mayoría de los encuestados acceden a estos contenidos, junto a la música y la ficción, a través del móvil, seguido del ordenador o portátil y de las Smart TV. 
Con respecto a las plataformas que usan para ver los contenidos, YouTube es la preferida por los jóvenes (99,10\%).

Este dato es coincidente con muchas de las indagaciones relacionadas. Y no solo sucede entre los jóvenes menores de edad, pues El Confidencial (11 de abril de 2017, online) publicaba hace dos años que "El 72\% de los jóvenes ve más Youtube que la televisión", titular usado en un artículo en el que se indicaba que "Para los encuestados la televisión tradicional tiene muchas cosas negativas, entre ellas, su programación, la nula flexibilidad para elegir los contenidos y la falta de interacción". Los datos del diario se basaban en un estudio de la web de tendencias Cultture, que realizó la encuesta a partir de una base de datos propia de usuarios españoles de entre 18 y 35 años, con 1.682 participantes. La tendencia, por tanto, ha sido observada también en sectores de edad no tan tempranos.

Sin embargo, no todas las tendencias conocidas se reproducen en nuestro estudio a pequeña escala. A diferencia de los datos publicados por la CNMC (2018), Movistar+ no ocupa la segunda posición en el ranking de nuestro estudio, ya que es reemplazada por Netflix, donde se emite una de las series más votadas por los menores (Stranger Things). En tercera posición estaría Movistar+ y a continuación HBO.

Como futura línea de estudio sería altamente interesante incluir en las investigaciones las posibilidades y usos de la nueva televisión de Apple. Sin embargo, no está entre las mencionadas por los menores que, sin embargo, indican otras plataformas o portales no tenidos en consideración porque no aparecen en los informes del sector, como son Plusdede, Masdede o Twitch TV. Además, cabe reflexionar sobre el hecho de que ninguno de los encuestados ha mencionado plataformas de contenido audiovisual creadas específicamente para su franja de edad, como es el caso de Televeo o de Playz, esta última la plataforma digital de TVE creada en 2017 para ofrecer contenidos interactivos y de producción exclusiva para la web.

Precisamente sobre la interacción de los jóvenes, Carlos A. Scolari (2018b) escribía recientemente en La Vanguardia una reflexión en la que indicaba que "En lugar de considerar a los jóvenes como consumidores abducidos por las pantallas, los interpelamos como prosumidores (productores + consumidores), personas capaces de generar y compartir contenidos de diferentes tipos y niveles de complejidad". Y muchos trabajos académicos apuntan hacia esta idea desde hace varios años, como los de Dezuanni y Hernández (2012), Vidales y Rubio (2014) o, muy recientemente, la interesante aproximación del ya mencionado Scolari (2018a).

No obstante, los resultados que aquí presentamos no son coincidentes con los anteriores, pues una de las asignaturas pendientes de los jóvenes es el grado de interacción. De hecho, con este estudio, se cuestiona el convencionalismo de que internet es el paradigma de la interactividad. Sólo una quinta parte de los encuestados afirmó participar activamente valorando o comentando los contenidos que visualizaban, mientras que el resto se limitaba a consumir los contenidos, imitando así el modelo de televisión lineal con un proceso clásico de comunicación emisor-receptor.

El papel de prosumidor no está consolidado entre estos jóvenes, ya que sólo el 35,14\% de los participantes había subido contenido audiovisual a internet, sobre todo en redes sociales como Instagram, Twitter o YouTube. Aquellos crean contenidos en redes sociales, no interactúan con los contenidos que consumen en las plataformas online, asumiendo el rol de un receptor pasivo.

Esta investigación, que constituye una primera aproximación al campo de estudio a través de la indagación en un caso concreto y geográficamente localizado de un centro, ofrece un punto de partida para ampliar los interrogantes y cues- 
tionamientos. A partir de las conclusiones esbozadas se dibujan diversas vías de continuación de la temática tratada. Por ejemplo, sería de gran interés que, a través del uso del cuestionario proporcionado por las autoras, se ampliasen las perspectivas con datos de alumnos de otros institutos, diversas franjas de edad y localidades diferentes, pudiendo establecerse también conclusiones que tuviesen en cuenta aspectos sociodemográficos como el género o la edad, y pudiendo incluir asimismo otras variables no contempladas en la concepción inicial de esta investigación. A todo ello se dedicarán los esfuerzos futuros.

\section{Referencias bibliográficas}

Adelantado, E. y Martí, J. (2011): “Contenidos audiovisuales y televisivos para dispositivos móviles: una aproximación al mercado español”. adComunica. Revista Científica de Estrategias, Tendencias e Innovación en Comunicación, 1, pp. 99-113. Disponible en: http://dx.doi.org/10.6035/2174-0992.2011.1.8 [Consultado el 22/10/2018].

Caldera-Serrano, J. y León-Moreno, J.A. (2017): “Plataformas de difusión de contenidos audiovisuales para telefonía móvil: realidad y potencialidad". Biblionline, João Pessoa, 13 (4), pp. 63-73.

Clares-Gavilán, J. y Medina-Cambrón, A. (2018): “Desarrollo y asentamiento del vídeo bajo demanda (VOD) en España: el caso de Filmin”. El profesional de la información, 27(4), pp. 909-920. Disponible en: https://doi.org/10.3145/epi.2018.jul.19 [Consultado el 14/10/2018].

CNMC (2018). Tres de cada diez hogares con Internet consumen contenidos audiovisuales online de pago. Disponible en: https://www.cnmc.es/node/368440 [Consultado el 19/10/2018].

De Mena Dávila, A. (2015). Televisión bajo demanda, ¿el fin de la televisión tradicional? Trabajo Fin de Grado. Universidad de Extremadura.

Dezuanni, M., y Hernández, A. M. (2012). Prosumidores interculturales: la creación de medios digitales globales entre los jóvenes. Comunicar: Revista científica iberoamericana de comunicación y educación, 38, 59-66. Disponible en: http://dx. doi.org/10.3916/C38-2012-02-06 [Consultado el 14/10/2018].

El Confidencial (11 de abril de 2017). El 72\% de los jóvenes ve más Youtube que la televisión. Disponible en: https://www. elconfidencial.com/television/2017-04-11/encuesta-mayoria-jovenes-prefiere-youtube-frente-television_1365435/[Consultado el 07/09/2018].

Galán Fajardo, E. y Del Pino Romero, C. (2009): “Jóvenes, ficción televisiva y nuevas tecnologías”. Área Abierta. Revista de comunicación audiovisual y publicitaria, (25), 3-3.

García, F. (1993). “La encuesta”. En García, M., Ibáñez, J. y Alvira, F. El análisis de la realidad social. Métodos y técnicas de investigación (pp. 141-170). Madrid: Alianza Universidad Textos.

Garza, V. (2017): “La mitad de los internautas españoles ve series de televisión online”. Kantar Media. Disponible en: https://es.kantar.com/media/tv/2017/julio-2017-datos-sobre-consumo-de-series-ante-el-estreno-de-juego-de-tronos/ [Consultado el 09/09/2018]. 
González Aldea, P. y López Vidales, N. (2011): “La generación digital ante un nuevo modelo de televisión: contenidos y soportes preferidos". Anàlisi: quaderns de comunicació i cultura, (44), pp. 31-48.

Guerrero Pérez, E. (2018): “La fuga de los millennials de la televisión lineal”. Revista Latina de Comunicación Social, 73, pp. 1231-1246. Disponible en: http://dx.doi.org/10.4185/RLCS-2018-1304 [Consultado el 27/09/2018].

Heredia, V. (2017): “Revolución Netflix: desafíos para la industria audiovisual”, Chasqui. Revista Latinoamericana de Comunicación, (135), pp. 275-295. Disponible en: https://dialnet.unirioja.es/descarga/articulo/6109989.pdf [Consultado el 29/09/2018].

Izquierdo Castillo, J. (2017): “¿Quién ve la televisión? Revisión del modelo generalista en el espacio mediático convergente”. Trípodos, 40, pp. 31-54. Disponible en: https://www.raco.cat/index.php/Tripodos/article/view/335048/425723 [Consultado el 19/10/2018].

Limelight Networks (2017). The State of online video 2017. Disponible en: https://img03.en25.com/Web/LLNW/\%7B66d0e66a-ac16-4cle-9e12-d8d845f2bddf\%7D_State_of_Online_Video_2017.pdf [Consultado el 08/10/2018].

Muñoz López, L. (Coord.) (2017). Informe Anual del Sector de los Contenidos Digitales en España 2017. Ministerio de Energía, Turismo y Agenda Digital. Disponible en: http://www.ontsi.red.es/ontsi/sites/ontsi/files/Informe\%20anual\%20 del\%20sector\%20de\%20los\%20Contenidos\%20Digitales\%20en\%20Espa\%C3\%B1a\%20\%28Edici\%C3\%B3n\%202017\%29. pdf [Consultado el 17/10/2018].

Onieva, Á. (17 de noviembre de 2017). "Netflix supera el millón de suscriptores en España y HBO irrumpe con fuerza”. Fotogramas. Disponible en: http://www.fotogramas.es/series-television/Netflix-HBO-Espana-datos-suscriptores-2017 [Consultado el 14/10/2018].

Ortega, F, González Ispierto, B. y Pérez Peláez, ME. (2015): “Audiencias en revolución, usos y consumos de las aplicaciones de los medios de comunicación en tabletas y teléfonos inteligentes". Revista Latina de Comunicación Social, 70, pp. 627651. Disponible en: http://dx.doi.org/10.4185/RLCS-2015-1063 [Consultado el 11/10/2018].

Peñafiel, C., Fernández de Arroyabe, A. y López, N. (2005): “La televisión ya no es la que era...: una perspectiva desde España”. Signo y Pensamiento, 24(47), pp. 121-134. Disponible en: http://www.redalyc.org/articulo.oa?id=86004709 [Consultado el 15/10/2018].

Quintas, N. y González, A. (2016): “Consumo televisivo y su medición en España: camino hacia las audiencias híbridas”. $E l$ profesional de la información, 25(3), pp. 376-383. Disponible en: https://doi.org/10.3145/epi.2016.may.07 [Consultado el $17 / 10 / 2018]$.

Rivera, R., Santos, D., Cabrera, V. y Docal, M.C. (2015). Consumo de pornografía on-line y off-line en adolescentes colombianos. Comunicar. Revista científica de educomunicación, 46(24), 37-45. Disponible en: https://doi.org/10.3916/C462016-04 [Consultado el 17/10/2018].

Sánchez-Labella Martín, I. (2015). New entertainment management. Migración de los contenidos infantiles en España: de la televisión a internet. ComHumanitas: Revista Científica de Comunicación, 6(1), 97-111. 
Nuevos hábitos de consumo audiovisual en menores: aproximación a su análisis mediante encuestas

Scolari, C. A. (2018a). Adolescentes, medios de comunicación y culturas colaborativas. Aprovechando las competencias transmedia de los jóvenes en el aula. EC H2020 Research and Innovation Actions: España. Disponible en: https://digital. fundacionceibal.edu.uy/jspui/handle/123456789/247 [Consultado el 17/10/2018].

Scolari, C. A. (2018b). ¿Qué hacen los adolescentes con los medios?. La Vanguardia.com.

Vega, C. (5 de junio de 2018). “La televisión sucumbe a Internet de la mano de los más jóvenes”. Libre Mercado. Disponible en: https://www.libremercado.com/2018-06-05/la-television-sucumbe-a-internet-de-la-mano-de-los-mas-jovenes-1276619918/ [Consultado el 09/10/2018].

Vidales, N. L., Aldea, P. G. y De la Viña, E. M. (2012): “Jóvenes y televisión en 2010: un cambio de hábitos”. Zer-Revista de Estudios de Comunicación, 16 (30), pp. 97-113.

Vidales, N. L. y Rubio, L. G. (2014). La democratización del proceso comunicativo en radio: los jóvenes prosumidores. Vivat Academia, 126, 31-53. 